\title{
FRC-QE: a robust and comparable 3D microscopy image quality metric for cleared organoids
}

\author{
Friedrich Preusser $\mathbb{D}^{1, \dagger}{ }^{1,}$ Natália dos Santos ${ }^{2, \dagger}{ }^{3}$ Jörg Contzen ${ }^{3}$, Harald Stachelscheid ${ }^{4}$, \\ Érico Tosoni Costa ${ }^{2}$, Philipp Mergenthaler (1) ${ }^{3,5,6, *}$ and Stephan Preibisch (1) ${ }^{1,7, *}$
}

${ }^{1}$ Berlin Institute for Medical Systems Biology (BIMSB), Max Delbrück Center for Molecular Medicine in the Helmholtz Association (MDC), Berlin 10115, Germany, ${ }^{2}$ Molecular Oncology Center, Hospital Sirio-Libanese, São Paulo, SP 01308-050, Brazil, ${ }^{3}$ Department of Experimental Neurology, Center for Stroke Research Berlin, Charité - Universitätsmedizin Berlin, Berlin 10117, Germany, ${ }^{4}$ Stem Cell Core Facility, Berlin Institute of Health at Charité - Universitätsmedizin Berlin, Berlin 13353, Germany, ${ }^{5}$ Department of Neurology, Charité - Universitätsmedizin Berlin, Berlin 10117, Germany, ${ }^{6}$ BIH Academy, Berlin Institute of Health at Charité -Universitätsmedizin Berlin, Berlin 10117, Germany and ${ }^{7}$ Janelia Research Campus, Howard Hughes Medical Institute, Ashburn, VA 20147, USA

*To whom correspondence should be addressed.

${ }^{\dagger}$ The authors wish it to be known that, in their opinion, the first two authors should be regarded as Joint First Authors.

Associate Editor: Peter Robinson

Received on October 20, 2020; revised on February 4, 2021; editorial decision on February 28, 2021; accepted on March 4, 2021

\begin{abstract} https://github.com/PreibischLab/FRC-QE.

\section{Introduction}

Three-dimensional (3D) organoids are a powerful tool for studying cellular processes in tissue-like structures, enabling in vitro experiments in an organ-specific context (Schutgens and Clevers, 2020). However, although it is essential for interpreting experiments, it remains challenging to capture cellular structures of entire organoids using 3D fluorescence microscopy due to the organoid's dense structure and opacity. Optical clearing methods (Spalteholz 1914; Ueda et al., 2020) provide a solution for fixed organoids. Nevertheless, optimizing clearing protocols for a given sample type and staining is challenging due to the plethora of available clearing and staining protocols. Importantly, quantitative measures for assessing image quality across cleared fluorescent samples are missing, making the process of identifying the best-suited protocol laborious and biased to the human observer. To fill this gap, we propose Fourier ring correlation quality estimation (FRC-QE) that provides a robust readout of image quality for 3D fluorescent microscopy samples (Fig. 1a).
\end{abstract}

Summary: Here, we propose Fourier ring correlation-based quality estimation (FRC-QE) as a new metric for automated image quality estimation in 3D fluorescence microscopy acquisitions of cleared organoids that yields comparable measurements across experimental replicates, clearing protocols and works for different microscopy modalities.

Availability and implementation: FRC-QE is written in ImgLib2/Java and provided as an easy-to-use and macro-scriptable plugin for Fiji. Code, documentation, sample images and further information can be found under

Contact: philipp.mergenthaler@charite.de or stephan.preibisch@mdc-berlin.de

Supplementary information: Supplementary data are available at Bioinformatics online.

\section{Method and implementation}

Fourier ring correlation (FRC) relies on two independent realizations of the same signal to measure image resolution in frequency space and was used for both electron (Heel, 1987; Saxton and Baumeister, 1982) and superresolution fluorescence microscopy (Banterle et al., 2013; Koho et al., 2019; Nieuwenhuizen et al., 2013). We previously extended FRC to standard 3D fluorescence microscopy (Hörl et al., 2019) by approximating the necessary independent observations from subsequent slices in the image stack of the same object combined with normalization to more distant slices to suppress artifacts, which was used for qualitative visualization of image quality. Here, we show that it can be used as a quantitative measurement across experiments by further adjusting (see Supplementary Materials and Methods and Supplementary Fig. S1) and validating this concept as a non-machine learning based method for no-reference image quality assessment (NR-IQA), which we call FRC-QE. Importantly, FRC-QE is designed specifically for 3D fluorescence microscopy and its score depends on the area and block size in which it is computed, the z-spacing, type of image content (e.g. nuclear stain) and the point spread function (PSF). These parameters should therefore be held constant during a series of comparisons. It is also important to note that the FRC-QE score represents arbitrary numbers that do not directly relate to an actual measurement of image resolution, but only allow for a relative comparison. FRC-QE is implemented in ImgLib2 (Pietzsch et al., 2012) and the core algorithm scales to terabyte-sized datasets (Hörl et al., 
(a)

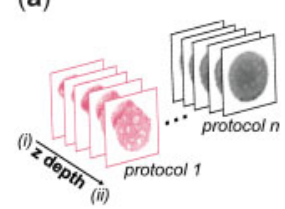

(c) Protocol 1 (ClearT2)

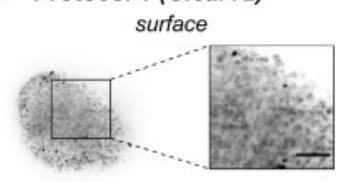

(d)

(d) Protocol 2 (Fructose-Glycerol)
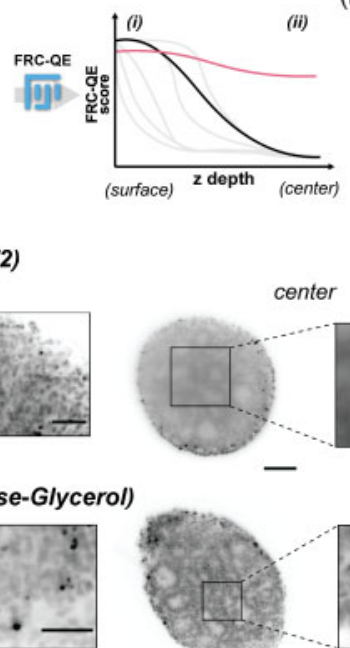

(b)
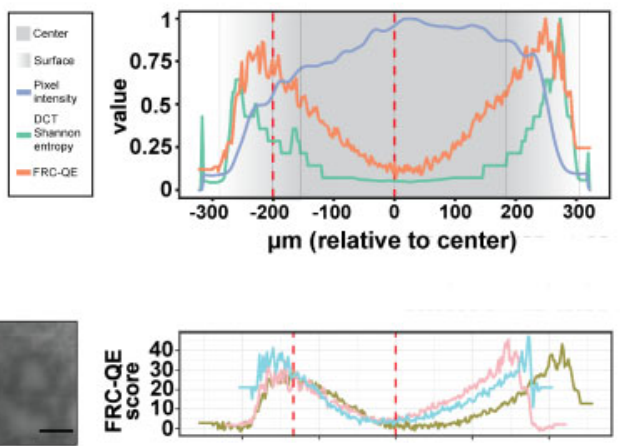

replicate $-1-2-3$

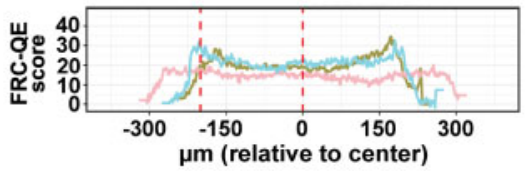

Fig. 1. Fourier ring correlation quality estimate (FRC-QE) for assessing clearing efficiency. (a) FRC-QE is designed to automatically quantify clearing efficiency across multiple protocols and is provided as a Fiji plug-in. (b) Comparison of image quality metrics for an insufficiently cleared organoid after light-sheet multi-view reconstruction. See Supplementary Figure S7 for a detailed comparison. (c,d) Image quality estimates for two different protocols with three replicates each, all imaged with light-sheet microscopy and multi-view reconstructed. Replicate 1 (light blue) always corresponds to the example images shown in the left panel. Dotted red lines correspond to example slices at the surface and within the center of the organoid. Scale bars correspond to 100 and $50 \mu \mathrm{m}$ for large panels and inlets, respectively

2019), while we also make it available to users as a macro-scriptable Fiji (Schindelin et al., 2012) plugin.

\section{Validation and results}

To validate FRC-QE, we generated human induced pluripotent stem-cell (hiPSC)-derived cerebral organoids of a defined size $(\sim 600 \mu \mathrm{m})$ that were stained with the nuclear dye Draq 5 and subsequently chemically fixed. We chose three straight-forward to implement clearing methods (Dekkers et al., 2019; Hama et al., 2011; Kuwajima et al., 2013) as proof of concept and applied them to our samples (Supplementary Fig. S2). Cleared organoids were imaged by multi-view light-sheet microscopy (Huisken et al., 2004) from opposite acquisition angles $\left(0^{\circ}\right.$ and $\left.180^{\circ}\right)$ with dual-illumination (left and right sided light-sheet illumination) and reconstructed computationally (Hörl et al., 2019) to significantly increase the volume of the sample imaged with high quality (Preibisch et al., 2010; Swoger et al., 2007). Due to the multi-view acquisition, image quality at the edges is expected to be higher than in the center of the organoid.

We first compared FRC-QE to the NR-IQA method DCT Shannon entropy, which was previously used in the context of automated microscopy (He and Huisken, 2020; Royer et al., 2016), and to plain image intensity that is commonly used to assess clearing quality (Kim et al., 2018; Matryba et al., 2019; Wan et al., 2018). FRC-QE and DCT Shannon recapitulate image quality throughout an organoid sample, while image intensity actually increases as quality decreases (Fig. 1 b and Supplementary Figs S3-S5).

We further validated FRC-QE for different microscopy modalities comparing Fructose-Glycerol-cleared organoids to uncleared organoids using a spinning-disk confocal microscope, where FRCQE recapitulated differences in image quality across image stacks and protocols (Supplemental Fig. S6).

Next, we used FRC-QE to identify the clearing protocol yielding the best image quality. Across all protocols nuclear structures can be easily visually identified at the surface of organoids. However, image quality differs at the center, with ClearT2 and ScaleA2 protocols resulting in blurred objects compared to the Fructose-Glycerol protocol (Fig. 1c,d and Supplementary Fig. S7), indicating differences in clearing efficiency between protocols as we performed them. While DCT Shannon entropy faithfully captured relative differences in clearing efficiency in one organoid (Fig. 1b), it is not suited for comparison between samples since it does not recapitulate the visually apparent differences in clearing efficiency that we observe in between protocols. In contrast, FRC-QE accurately recapitulates these differences (Fig. 1c,d and Supplementary Fig. S7) and shows that only Fructose-Glycerol-cleared samples retain constant quality throughout entire volumes of cleared organoids.

We additionally compared FRC-QE to the established machine learning based NR-IQA algorithm BRISQUE (Supplementary Fig. S8). However, machine learning algorithms have a training phase in which they learn important characteristics about the images. These data are usually not available for biological images that differ substantially depending on the sample and staining used. Therefore, new training data needs to be created for a new type of experiment, which is often infeasible, or training is performed on natural images instead. Overall, for our data a pre-trained BRISQUE performed similarly to FRC-QE, but locally shows unexpected behavior for certain images presumably due to training on a different type of sample image. In summary, an engineered metric like FRC-QE is expected to perform more predictably in previously unseen samples and is, we believe, well-suited for biological images.

\section{Conclusion}

We introduce FRC-QE, implemented in ImgLib2 (Pietzsch et al., 2012) and provided as Fiji (Schindelin et al., 2012) plugin, as a new non-machine learning based NR-IQA metric to automatically assess clearing efficiency from 3D fluorescence microscopy images. FRCQE can be applied to image data from different microscopy modalities, is comparable across protocols, and can therefore be used to identify the most suitable clearing protocol, which is often the one that achieves the necessary image quality to gain a certain insight given the lowest effort and cost. Overall, we believe that image quality estimation using FRC-QE will facilitate and significantly ease the process of choosing the right clearing protocol for a given biological sample. Furthermore, FRC-QE represents a promising approach for other automated image quality tasks in fluorescence microscopy.

\section{Acknowledgements}

The authors thank EU-Life and FAPESP (specifically Marta D. Agostinho and Anamaria Camargo) for initiating this collaboration during the FAPESP/EULIFE Symposium in 2016. They also thank Andrew Woehler, Nikita 
Vladimirov, Adam Taylor and Sreenivas Bhattiprolu for discussions and support during the project, Loïc Royer for sharing the DCT Shannon entropy implementation openly.

\section{Funding}

This work was supported by the Bundesministerium für Bildung und Forschung, Germany [BMBF, grant no. 031L0142A to S.P. and P.M., and in part by grants no. 16GW0191 and NUM-COVID 19 - Organo-Strat 01KX2021 to P.M. and H.S.] and HFSP [RGP0021/2018-102 to S.P. and F.P.]. F.P. was funded by a PhD fellowship from Studienstiftung des deutschen Volkes. S.P. was funded by MDC Berlin and HHMI Janelia. N.S. was funded by FAPESP's program of Research Internships Abroad (BEPE, 2019/ 06305-4) during her visit to the Preibisch lab at the MDC Berlin. P.M. was supported by the BIH-Charite Clinical Scientist Program funded by Charite Universitätsmedizin Berlin and the Berlin Institute of Health. E.T.C. was funded by the Hospital Sirio-Libanese/HSL/SBSHSL. Establishment of the Charité High Content Shared Facility was supported by the Deutsche Forschungsgemeinschaft [DFG, grant no. INST 335/591-1].

Conflict of Interest: none declared.

\section{References}

Banterle,N. et al. (2013) Fourier ring correlation as a resolution criterion for super-resolution microscopy. J. Struct. Biol., 183, 363-367.

Dekkers,J.F. et al. (2019) High-resolution 3D imaging of fixed and cleared organoids. Nat. Protoc., 14, 1756-1771.

Hama,H. et al. (2011) Scale: a chemical approach for fluorescence imaging and reconstruction of transparent mouse brain. Nat. Neurosci., 14, 1481-1488.

He,J. and Huisken,J. (2020) Image quality guided smart rotation improves coverage in microscopy. Nat. Commun., 11, 150.

Heel,M.V. (1987) Similarity measures between images. Ultramicroscopy, 21, 95-100.

Hörl,D. et al. (2019) BigStitcher: reconstructing high-resolution image datasets of cleared and expanded samples. Nat. Methods, 16, 870-874.
Huisken,J. et al. (2004) Optical sectioning deep inside live embryos by selective plane illumination microscopy. Science, 305, 1007-1009.

Kim,J.H. et al. (2018) Optimizing tissue-clearing conditions based on analysis of the critical factors affecting tissue-clearing procedures. Sci. Rep., 8 , 12815 .

Koho,S. et al. (2019) Fourier ring correlation simplifies image restoration in fluorescence microscopy. Nat. Commun., 10, 3103.

Kuwajima,T. et al. (2013) ClearT: a detergent- and solvent-free clearing method for neuronal and non-neuronal tissue. Development, 140, 1364-1368

Matryba,P. et al. (2019) Advances in ex situ tissue optical clearing. Laser Photonics Rev., 13, 1800292.

Nieuwenhuizen,R.P.J. et al. (2013) Measuring image resolution in optical nanoscopy. Nat. Methods, 10, 557-562.

Pietzsch,T. et al. (2012) ImgLib2-generic image processing in Java. Bioinformatics, 28, 3009-3011.

Preibisch,S. et al. (2010) Software for bead-based registration of selective plane illumination microscopy data. Nat. Methods, 7, 418-419.

Royer,L.A. et al. (2016) Adaptive light-sheet microscopy for long-term, high-resolution imaging in living organisms. Nat. Biotechnol., 34, 1267-1278.

Saxton,W.O. and Baumeister,W. (1982) The correlation averaging of a regularly arranged bacterial cell envelope protein. J. Microscopy, 127, 127-138.

Schindelin,J. et al. (2012) Fiji: an open-source platform for biological-image analysis. Nat. Methods, 9, 676-682.

Schutgens,F. and Clevers,H. (2020) Human organoids: tools for understanding biology and treating diseases. Annu. Rev. Pathol. Mech. Dis., 15, 211-234.

Spalteholz,W. (1914) Über das Durchsichtigmachen von menschlichen und tierischen Präparaten und seine theoretischen Bedingungen, nebst Anhang: Über Knochenfärbung. Hirzel, Leipzig.

Swoger,J. et al. (2007) Multi-view image fusion improves resolution in three-dimensional microscopy. Opt. Express, 15, 8029-8042.

Ueda,H.R. et al. (2020) Whole-brain profiling of cells and circuits in mammals by tissue clearing and light-sheet microscopy. Neuron, 106, 369-387.

Wan,P. et al. (2018) Evaluation of seven optical clearing methods in mouse brain. Neurophotonics, 5, 035007 